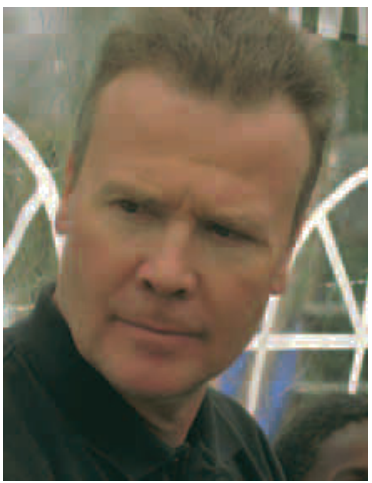

G. Dine

\section{Vous avez dit} Nanobiotechnologies et médecine Pré-emptive?!

Le grand public et parfois même les professionnels concernés commencent à peine à digérer la signification du terme biotechnologie dont les impacts se multiplient autour de nous notamment dans le domaine de la biologie clinique qu'émerge un nouveau barbarisme la nanomédecine. La dimension nanométrique a une trentaine d'années. Elle s'appuie sur la possibilité qu'ont offerte les physiciens à disposer de systèmes de mesure ou d'information à l'échelle de l'atome c'est-à-dire celle du nanomètre et de l'Angström. Les applications ont été très rapides en particulier dans le domaine de l'électronique et de la fabrication de matériaux utiles en informatique. Les nanotechnologies sont aujourd'hui employées dans le développement d'autres matériaux ainsi le traitement de surface, des prothèses humaines mais aussi de nombreuses applications dans les secteurs aérospatiaux et automobiles. La progression des outils à pu rendre la matière vivante accessible et permettre par conséquent l'apparition des nanobiotechnologies. En quelques années les systèmes analytiques de recherche ont pu être adaptés à l'exploration des biomolécules. Parallèlement l'échelle nanométrique est devenue une réalité pour la conception de puces à ADN, de puces à protéines, et les dispositifs labs on chips où on fait appel à la nanofluidique qui ne relève pas des lois classiques de la mécanique des fluides. La progression dans le domaine médical et biologique est excessivement rapide en raison des aspects stratégiques inhérents à ces technologies. Plusieurs opérateurs associés au monde de la biologie clinique et de la médecine se manifestent comme le CEA à travers le LETI qui est implanté sur Grenoble. Aujourd'hui les nanobiotechnologies nous interpellent. Les fantastiques progrès attendus sont sources d'espoirs face aux pathologies graves. Mais les nanotechnologies posent de nombreuses questions d'ordre social, éthique, économique et politique. Le coût du développement ne peut être envisagé que dans un contexte qui associe sciences académiques et financements industriels. Le risque toxicologique doit être impérativement appréhendé d'une façon préventive pour éviter des dérapages lourds de conséquence. La relation aux utilisateurs ne peut pas être éludée dans ces conditions. Confrontée à ces questionnements la Communauté Européenne a crée en 2009 une plate forme d'action associant tous les protagonistes. C'est dans ce contexte que I'INCA a organisé en octobre 2009 le colloque Onco Nano, afin d'établir une forme d'état de l'art. La nanomédecine peut être utilisée dans de nombreuses disciplines mais l'oncologie est probablement une cible de choix. Passer du statut de médecine symptomatique qui est notre quotidien actuel au concept de médecine pré-emptive est un challenge qui mérite réflexion et attention pour les actions à mener et à venir. Pour ces raisons Biotribune Magazine a choisi de s'associer à l'INCA afin de porter témoignage grâce aux articles publiés dans ce dernier numéro de l'année 2009.

G. Dine 